\title{
Estimación de la riqueza de coleópteros epigeos de la Reserva Nacional Pingüino de Humboldt (Regiones de Atacama y Coquimbo, Chile)
}

\section{Estimation of the richness of epigean coleopterans of the Pingüino of Humboldt National Reserve (Atacama And Coquimbo Regions, Chile)}

\author{
Fermín M. Alfaro* \& Jaime Pizarro-Araya
}

Laboratorio de Entomología Ecológica, Departamento de Biología, Universidad de La Serena, Casilla 554, Chile. Instituto de Investigación Multidisciplinar en Ciencia y Tecnología, Universidad de La Serena, Casilla 554, La Serena, Chile. *E-mail: fmalfaro@userena.cl

\begin{abstract}
The estimate of the richness is an essential element in the characterization of biodiversity, so that regional inventories of certain groups of taxa as epigean coleopterans are important for planning conservation efforts in protected natural areas. By using accumulation curves and non-parametric estimators, the quality of various samples on estimating the richness of epigean coleopterans of the Pingüino Humboldt National Reserve (Atacama and Coquimbo Regions, Chile) was evaluated. The asymptotic models tested showed theoretical species richness close to that observed in the Choros and Damas islands, however in Chañaral Island no clear asymptote was observed, suggesting an incomplete inventory. All estimators evaluated showed richness values greater to the observed values for all the studied islands, however its accumulation curves did not stabilize in any island except for ICE and Jack 2 in Choros and Damas. We propose to conduct additional sampling in particular habitats of this reserve, as well as to include other seasons of the year and different years in order to register rare species not recorded to date by our sampling.
\end{abstract}

KeYwords: Biodiversity, Coleoptera, Conservation, Islands, Species richness estimation

\begin{abstract}
RESUMEN
La estimación de la riqueza constituye un elemento esencial en la caracterización de la biodiversidad tanto a nivel local como regional. Evaluar el grado de completitud de los inventarios de riqueza locales es una tarea clave para dirigir de manera eficaz los esfuerzos de muestreo en áreas naturales protegidas. Mediante el uso de curvas de acumulación y estimadores no paramétricos, evaluamos la calidad de diversos muestreos sobre la estimación de la riqueza de coleópteros epigeos de la Reserva Nacional Pingüino de Humboldt (Regiones de Atacama y Coquimbo, Chile). Los modelos asintóticos evaluados mostraron una riqueza teórica de especies cercana a la observada en las islas Choros y Damas; sin embargo, para Isla Chañaral no se observó una asíntota clara, lo que sugiere un inventario incompleto. Todos los estimadores no paramétricos evaluados mostraron valores de riqueza sobre lo observado para todas las islas estudiadas; sin embargo, sus curvas de acumulación no se estabilizaron en ninguna isla a excepción de ICE y Jack 2 en Choros y Damas. Nosotros proponemos dirigir muestreos adicionales en hábitats particulares de esta reserva, así como también incluir otras estaciones del año y diferentes años con el objetivo de registrar especies raras no registradas a la fecha por nuestros muestreos.
\end{abstract}

Palabras clave: Biodiversidad, Coleoptera, Conservación, Islas, Estimación de la Riqueza de Especies

\section{INTRODUCCIÓN}

La riqueza constituye una forma directa y simple de cuantificar la biodiversidad ya que puede estimar el número total de especies que habitan un área particular (Magurran 1988, 2004). Sin embargo, la cuantificación de este parámetro presenta cierta complejidad ya que se hace imposible registrar la totalidad de especies que se distribuyen en un área determinada (Gotelli \& Colwell 2001; JiménezValverde \& Hortal 2003). Algunas complicaciones en la estimación de la riqueza es su sensibilidad en el número de individuos recolectados y el número, tamaño y disposición espacial de las unidades muestrales (Magurran 2004; Gotelli \& Colwell 2011). Por esta razón el uso de técnicas 
que estimen la riqueza se ha convertido en una herramienta muy utilizada para estimar el potencial número de especies a partir de diferentes muestreos sistemáticos (Palmer 1990; Colwell \& Coddington 1994). Una de las herramientas más utilizadas para esto son las curvas de acumulación, que permiten estimar el número total de especies de un área mediante la extrapolación de la asíntota de una función (Soberón \& Llorente 1993; Gotelli \& Colwell 2001). Otros métodos como el índice de distinción taxonómica basado en matrices de presencia/ausencia es independiente del tamaño muestral y del esfuerzo de muestreo (Clarke \& Warwick 1998). Sin embargo, existen otros métodos como los estimadores paramétricos, los cuales ajustan la abundancia relativa de las especies en una muestra a diferentes modelos matemáticos paramétricos (e.g. serie logarítmica, distribución log-normal, modelo de vara quebrada) (Moreno 2001) y los estimadores no paramétricos basados en la distribución de especies raras (Colwell \& Coddington 1994; González-Oreja et al. 2010; Magurran 2004). Estos últimos presentan la ventaja de poseer un sesgo menor en comparación a la extrapolación de las curvas de acumulación (Colwell \& Coddington 1994; Gotelli \& Colwell 2001) y requieren menor cantidad de datos que los necesarios para el uso de métodos paramétricos (Brose 2002). Estas estimaciones relacionan el número de especies con el esfuerzo de muestreo llevado a cabo en un área particular y representa una importante herramienta a la hora de evaluar la calidad y completitud de los inventarios de la biodiversidad (Sánchez-Fernández et al. 2011; GómezAnaya et al. 2014). La importancia de la estimación de la riqueza radica en que constituye un criterio para definir áreas de alta riqueza de especies y por tanto prioritarias a la hora de ejecutar planes de conservación y manejo de áreas protegidas (Cutko 2009; Fattorini 2013a).

La evaluación de los inventarios de la biodiversidad se hace cada vez más importante cuando los esfuerzos de muestreos se concentran en áreas naturales protegidas, las que no siempre se pueden estudiar de forma completa y sistemática. Este es el caso de la Reserva Nacional Pingüino de Humboldt, localizada en el borde costero de Punta Choros entre las regiones de Atacama y Coquimbo (Chile) y con una superficie de 859,3 ha (CONAF 1997). Esta área pertenece al SNASPE (Sistema Nacional de Áreas Silvestres Protegidas del Estado) y está formada por las islas Choros, Damas y Chañaral (CONAF 1997). Diversos estudios de artrópodos se han realizado en estos sistemas insulares, entre ellos la descripción de especies endémicas (PizarroAraya \& Flores 2004; Ojanguren-Affilastro et al. 2007; Grismado \& Pizarro-Araya 2016), estudios ecológicos sobre la estructura comunitaria de insectos epigeos (Alfaro et al. 2009, 2014, 2016; Pizarro-Araya et al. 2014a), Scorpiones (Arachnida) (Pizarro-Araya et al. 2014b), estudios de las variaciones morfológicas de coleópteros tenebriónidos (Benítez et al. 2014), caracterización de las comunidades de Solifugae (Arachnida) en islas y áreas continentales adyacentes (Valdivia et al. 2008, 2011) y evaluación de estados de conservación (Pizarro-Araya et al. 2012; PizarroAraya et al. 2017). A la fecha no se dispone de estudios que evalúen los niveles de riqueza y los esfuerzos de muestreo de artrópodos de áreas insulares. Considerando que existen muestreos estacionales sistemáticos dirigidos a este grupo taxonómico y en particular a los coleópteros epigeos de la Reserva Nacional Pingüino de Humboldt nosotros planteamos las siguientes preguntas: (1) ¿cuál es el nivel de esfuerzo de los muestreos de coleópteros epigeos realizados en la Reserva Nacional Pingüino de Humboldt? y (2) ¿cuál es la magnitud necesaria de estos muestreos para completar la riqueza total esperada para este grupo taxonómico? Mediante el uso de curvas de acumulación y estimadores no paramétricos evaluamos la calidad y completitud de un conjunto de muestreos estacionales de coleópteros epigeos realizados en diferentes años e islas en un ecosistema insular costero desértico del norte de Chile.

\section{MATERIALES Y MÉTODOS}

Área DE ESTUdio

El estudio se realizó en la Reserva Nacional Pingüino de Humboldt, ubicada en el límite costero de las provincias de Huasco (Región de Atacama) y Elqui (Región de Coquimbo). Esta área protegida está formada por las islas Choros y Damas (Región de Coquimbo) con una superficie de 322 y 56 ha respectivamente y Chañaral (Región de Atacama) con una superficie de 507,3 ha (CONAF 1997) (Fig. 1).

El clima del área de estudio presenta tendencia mediterránea (di Castri \& Hajek 1976). Las temperaturas son bajas al igual que la amplitud térmica diaria y anual producto de la influencia marina (Armesto et al. 1993). La precipitación anual promedio del área es $\sim 90 \mathrm{~mm}$; con años secos y lluviosos que ocurren en ciclos irregulares relacionados al evento El Niño Oscilación del Sur (ENOS) (Novoa \& Villaseca 1989). Los antecedentes geomorfológicos y biofísicos de parte del área de estudio se describen en Aguirre (1967) y Castro \& Brignardello (2005). La diversidad florística del área se encuentra detallada en Arancio \& Jara (2007).

Metodología, diseño y tamaño de MUeStReo

Los muestreos se realizaron en diferentes hábitats de las islas Chañaral, Choros y Damas. Se utilizaron muestreos provenientes de tres hábitats distintos, en términos vegetacionales, en cada isla; tanto para Choros y Damas (ver Alfaro et al. 2016) como para Chañaral (ver Pizarro-Araya et al. 2014a). En cada uno de los hábitats descritos arriba se instalaron dos parcelas de 4 × $5 \mathrm{~m}$ las que consistieron en un conjunto de 20 trampas de intercepción cada una sensu Cepeda-Pizarro et al. (2005a, 2005b). Cada trampa 


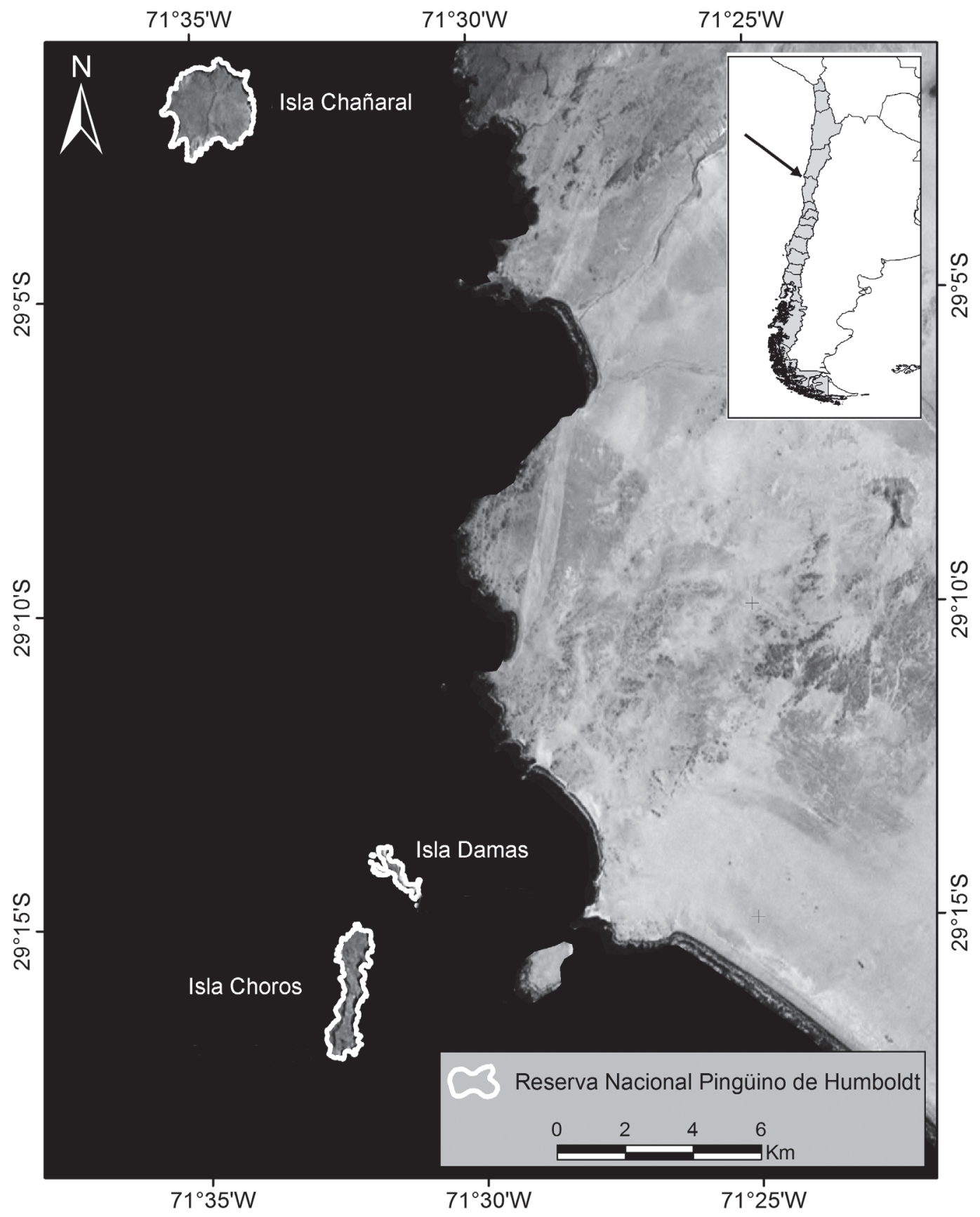

Figura 1. Localización geográfica de la Reserva Nacional Pingüino de Humboldt (Regiones de Atacama y Coquimbo). / Geographical location of the Pingüino de Humboldt National Reserve (Atacama and Coquimbo Regions, Chile).

consistió en 2 vasos plásticos dispuestos uno sobre el otro, con el vaso interno de fácil remoción $(7,4 \mathrm{~cm}$ de diámetro $\mathrm{x}$ $10,2 \mathrm{~cm}$ de alto), el que fue llenado hasta dos tercios de su capacidad con una mezcla de agua $(80 \%)$, etanol $(10 \%)$ y detergente doméstico $(10 \%)$. Las trampas operaron tres días durante cada uno de los tres meses o fechas (agosto, octubre, diciembre) de 2006 en las islas Choros y Damas, mientras que en Isla Chañaral operaron el mismo número de días durante los meses de noviembre de 2012 y enero de 2013.
El material capturado fue retirado, limpiado y conservado en alcohol (70\%) hasta el momento de su procesamiento y montaje. Para la identificación taxonómica de coleópteros epigeos se siguió a Kulzer (1959), Peña (1971, 1974, 1980), Solervicens (2001), Elgueta \& Marvaldi (2006), PizarroAraya \& Flores (2004), Flores \& Pizarro-Araya (2012, 2014). El material colectado está depositado en la colección del Laboratorio de Entomología Ecológica, Universidad de La Serena, Chile (LEULS). 
De acuerdo a Moreno \& Halffter (2000) el esfuerzo de muestreo se cuantificó por medio de unidades de muestreo, las que correspondieron a cada una de las parcelas descritas arriba. Tanto para Choros como Damas se analizaron 18 unidades de muestreo, las que correspondieron al total proveniente de dos parcelas en tres hábitats por tres fechas. Asimismo, para Isla Chañaral se analizaron 14 unidades de muestreo, las que correspondieron al total proveniente de dos parcelas en tres hábitats por dos fechas más dos unidades de muestreo adicionales provenientes de la última fecha de muestreo (enero 2013).

\section{ANÁLISIS DE DATOS}

Para evaluar la relación entre el esfuerzo de muestreo (unidades de muestreo) y el número de especies observado, se construyeron curvas de acumulación de especies mediante las funciones de los modelos asintóticos de Clench y dependencia lineal (Soberón \& Llorente 1993), los cuales corresponden a estimadores paramétricos que requieren muestras aleatorias, independencia de las observaciones y que los datos se distribuyan de cierta forma (Escalante 2005). El modelo de Clench se deriva de la ecuación de Michaelis Menten y estima la probabilidad de encontrar nuevas especies conforme se incrementa el esfuerzo de muestreo en campo (Clench 1979). Por otra parte, el modelo de dependencia lineal considera que la función de recolección de nuevas especies depende linealmente del tamaño total de muestras y posee parámetros constantes en el tiempo. Este modelo es adecuado cuando se muestrean áreas relativamente pequeñas, taxa bien conocidos o ambos. Tanto el modelo de Clench como el de dependencia lineal permiten calcular el esfuerzo necesario para alcanzar una determinada proporción de la biota (Soberón \& Llorente 1993). Estas funciones fueron ajustadas por medio de la estimación no lineal del algoritmo de Simplex \& QuasiNewton siguiendo a Jiménez-Valverde \& Hortal (2003) en el programa Statistica ver. 10 (Statsoft 2011). Para eliminar el efecto del orden en que se adicionan las muestras a la curva se usaron 100 aleatorizaciones con reemplazamiento siguiendo a Walther \& Moore (2005) y Colwell (2013), mediante el programa EstimateS ver. 9.1.0 (Colwell 2013).

Para estimar la riqueza se calcularon las curvas suavizadas de acumulación de especies de acuerdo a los siguientes estimadores no paramétricos: Chao 1, Chao 2, los estimadores de cobertura basados en abundancia (ACE) e incidencia (ICE), jackknife de primer y segundo orden (Jack 1 y Jack 2 respectivamente) y bootstrap (Chazdon et al. 1998; Colwell 2013). Chao 1 se basa en el número de especies raras en la muestra, mientras que Chao 2 considera las especies observadas en exactamente una y dos unidades de muestreo. ACE se basa en abundancias de aquellas especies con diez o menos individuos en la muestra, mientras que ICE se basa en las especies raras presentes en diez o menos unidades de muestreo. Jack 1 considera el número de especies presentes en sólo una unidad de muestreo, mientras que Jack 2 toma en cuenta las especies presentes en dos unidades de muestreo. Finalmente el estimador bootstrap se basa en la proporción de unidades de muestreo que contienen a cada especie.

El empleo de estimadores no paramétricos o de "distribución libre" no asume distribuciones particulares ni supuestos requeridos por los estimadores paramétricos; su cálculo es sencillo y son relativamente efectivos (Escalante 2003). Estos estimadores son universalmente válidos para cualquier distribución de abundancia de especies y más robustos que los estimadores paramétricos basados en modelos paramétricos de abundancia específica (Chao \& Chiu 2016). Todos los estimadores fueron calculados mediante el programa EstimateS ver. 9.1.0 (Colwell 2013). Posteriormente los estimadores fueron evaluados en relación a su sesgo y exactitud, mediante la relación de la riqueza estimada ( $\left.\mathrm{S}_{\text {estimada }}\right)$ y la riqueza verdadera $\left(\mathrm{S}_{\text {verdadera }}\right)$. Valores positivos y negativos del sesgo indican sobrestimación y subestimación de la riqueza respectivamente, mientras que valores cercanos a cero de la exactitud indican mayor cercanía de la riqueza estimada a la riqueza verdadera (Sokal \& Rohlf 1995). Para la estimación de estos parámetros se siguió a Chiarucci et al. (2003) y Brose et al. (2003).

\section{RESULTADOS}

Los datos presentaron un buen ajuste para las funciones de Clench y dependencia lineal. El modelo de dependencia lineal explicó en mayor medida el comportamiento de los datos para las islas Choros $\left(R^{2}=0,9961\right)$ y Damas $\left(R^{2}=\right.$ 0,9998), mientras que el modelo de Clench lo hizo para Isla Chañaral $\left(R^{2}=0,9996\right)$ (Tabla 1).

El modelo de dependencia lineal estimó un número teórico de especies cercano al observado en las islas Choros y Damas y mostró un porcentaje de eficiencia de muestreo de $101,0 \%$ y $98,9 \%$ respectivamente. Por su parte el modelo de Clench estimó un mayor número de especies y mostró un 75,6\% de eficiencia de muestreo en Isla Chañaral (Tabla 1). Sólo las curvas de las islas Choros y Damas mostraron una tendencia a estabilizarse, mientras que la curva para Isla Chañaral no alcanzó una asíntota definida (Fig. 2).

Todos los estimadores no paramétricos mostraron valores de riqueza estimada por encima de la riqueza observada en todas las islas (Tabla 1, Figs. 3, 4, 5). Las curvas de acumulación de especies no alcanzaron una asíntota definida excepto ICE y Jack 2 en Choros y Damas. Los estimadores ACE, ICE, Chao 2 y Jack 2 mostraron un crecimiento inicial alto (Figs. 3, 4, 5). Chao 1 mostró una curva similar a la riqueza observada tanto en Isla Damas como en Chañaral, mientras que Bootstrap sólo lo hizo en Chañaral. Estas curvas estuvieron ligeramente por encima de la riqueza observada (Figs. 3, 4, 5). 


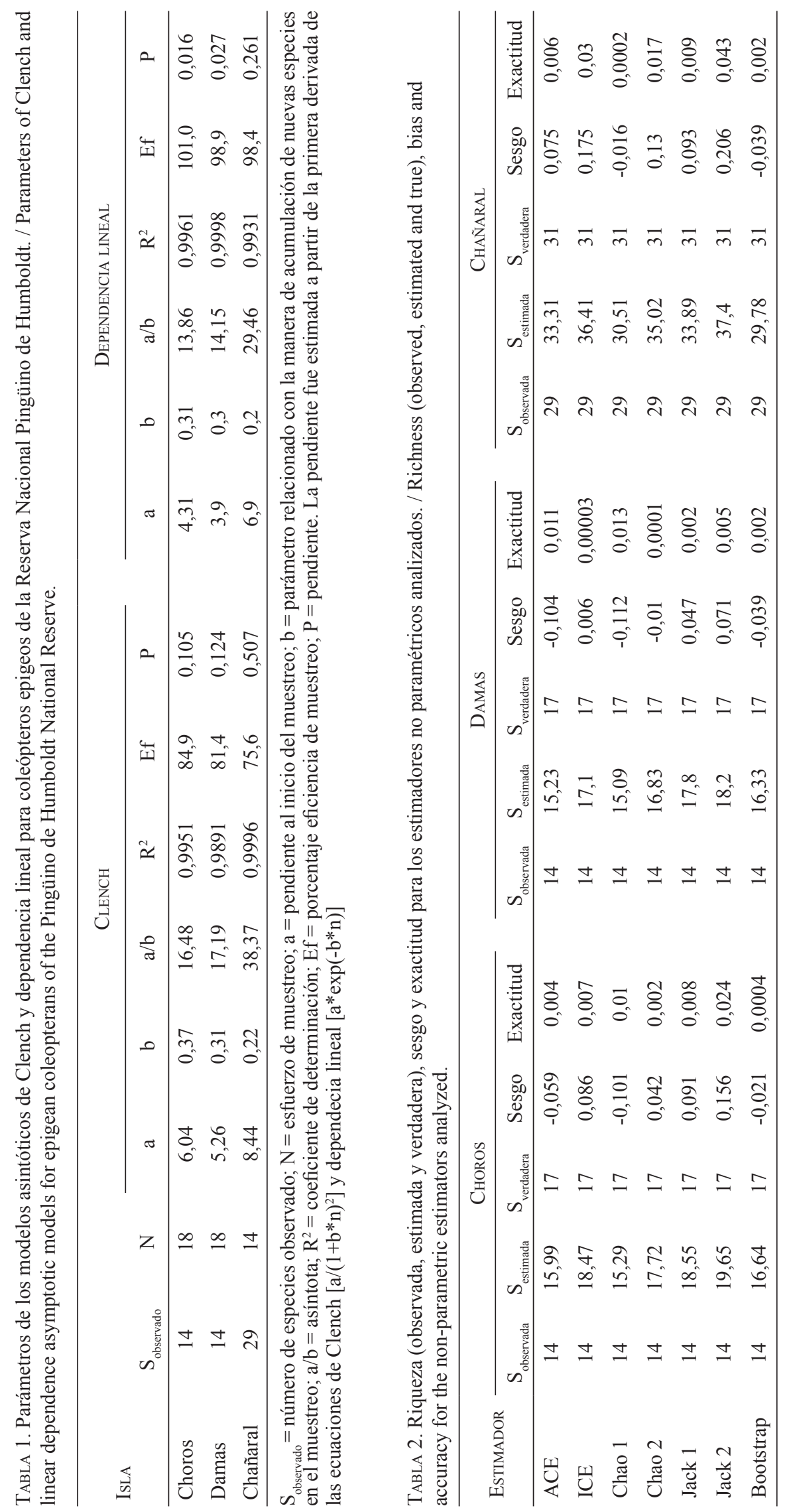


En términos de sesgo y exactitud Chao 2 e ICE fueron los estimadores menos sesgados y de mayor exactitud para Choros y Damas respectivamente, mostrando sus curvas de acumulación un crecimiento inicial rápido; sin embargo, sólo la curva de acumulación de ICE se estabilizó después de agruparse 9 muestreos en Isla Choros, mientras que para Isla Damas las curvas de acumulación de ICE y Jack
2 se estabilizaron luego de agrupar 8 muestreos (Figs. 3, 4). Los estimadores menos sesgados para Chañaral fueron ACE y Jack 1. En términos de exactitud los estimadores que presentaron valores de riqueza estimada más cercanos a la riqueza verdadera fueron Bootstrap y Chao 2 para Choros, ICE y Chao 2 para Damas y Chao 1 y Bootstrap para Chañaral (Tabla 2).
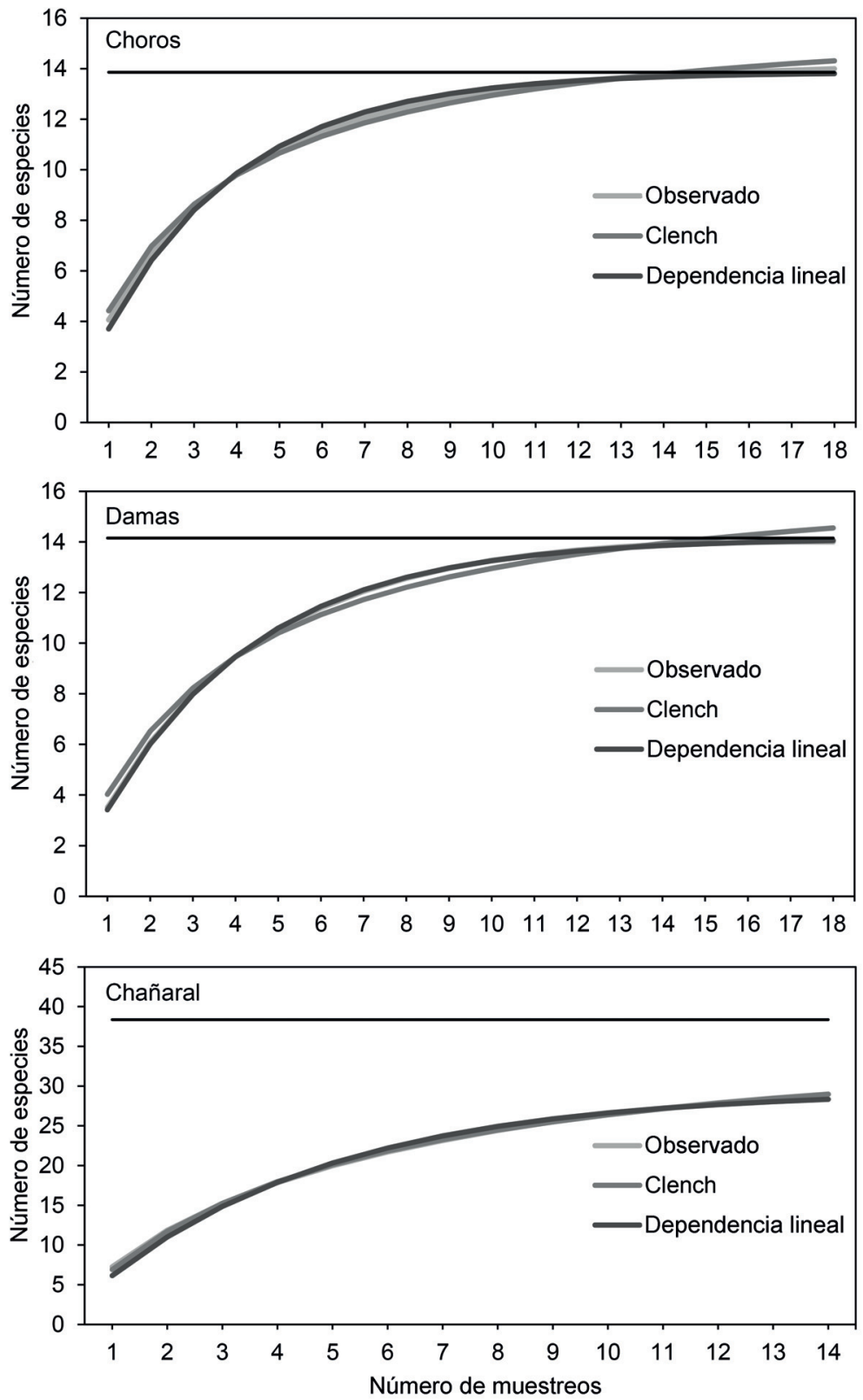

Figura 2. Curvas suavizadas de acumulación de especies según las funciones de Clench y dependencia lineal para coleópteros epigeos de la Reserva Nacional Pingüino de Humboldt. La línea horizontal superior indica el valor de riqueza asintótica de acuerdo a la mejor función de acumulación de especies. / Smooth richness accumulation curves according Clench and linear dependence functions for epigean coleopterans of the Pingüino de Humboldt National National Reserve. The upper horizontal line indicates the asymptotic richness value estimated according to the best species accumulation function. 

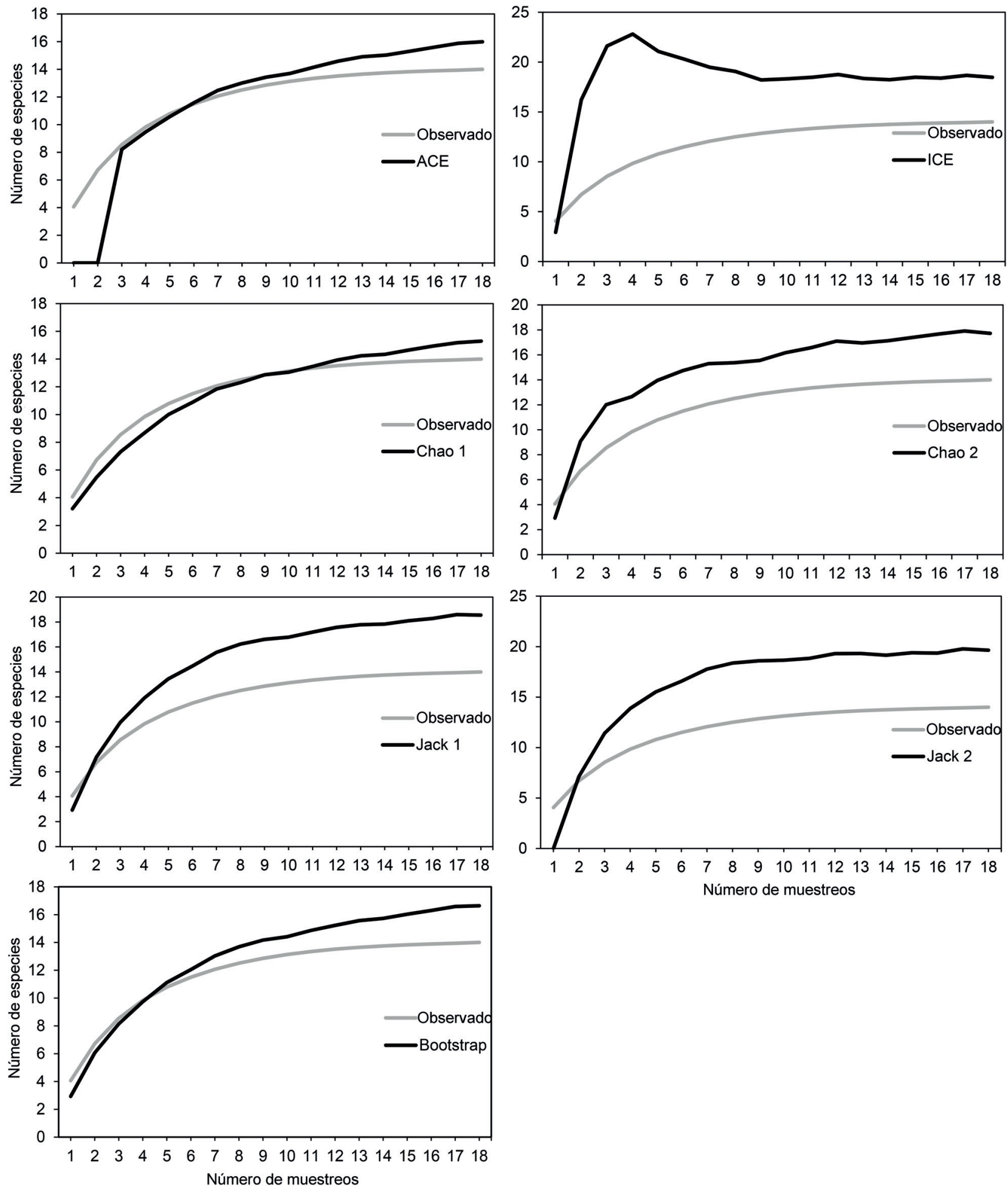

FIgURA 3. Curvas suavizadas de acumulación de especies de acuerdo a los estimadores no paramétricos ACE, ICE, Chao 1, Chao 2, Jack 1, Jack 2 y Bootstrap, para coleópteros epigeos de Isla Choros. / Smooth richness accumulation curves for the non-parametric estimators ACE, ICE, Chao 1, Chao 2, Jack 1, Jack 2 and Bootstrap, for epigean coleopterans from Choros Island. 

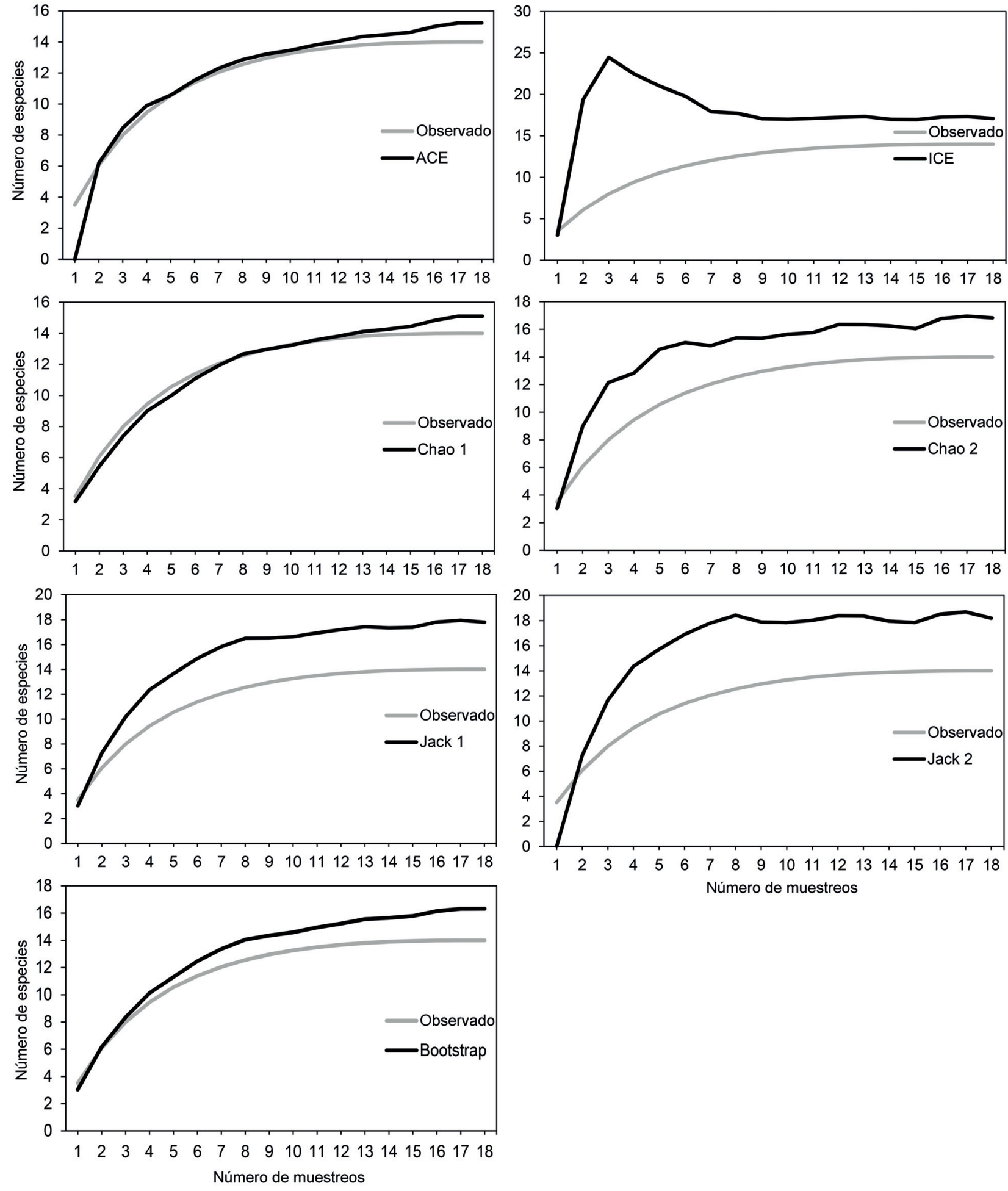

Figura 4. Curvas suavizadas de acumulación de especies de acuerdo a los estimadores no paramétricos ACE, ICE, Chao 1, Chao 2, Jack 1, Jack 2 y Bootstrap, para coleópteros epigeos de Isla Damas. / Smooth richness accumulation curves for the non-parametric estimators ACE, ICE, Chao 1, Chao 2, Jack 1, Jack 2 and Bootstrap, for epigean coleopterans from Damas Island. 

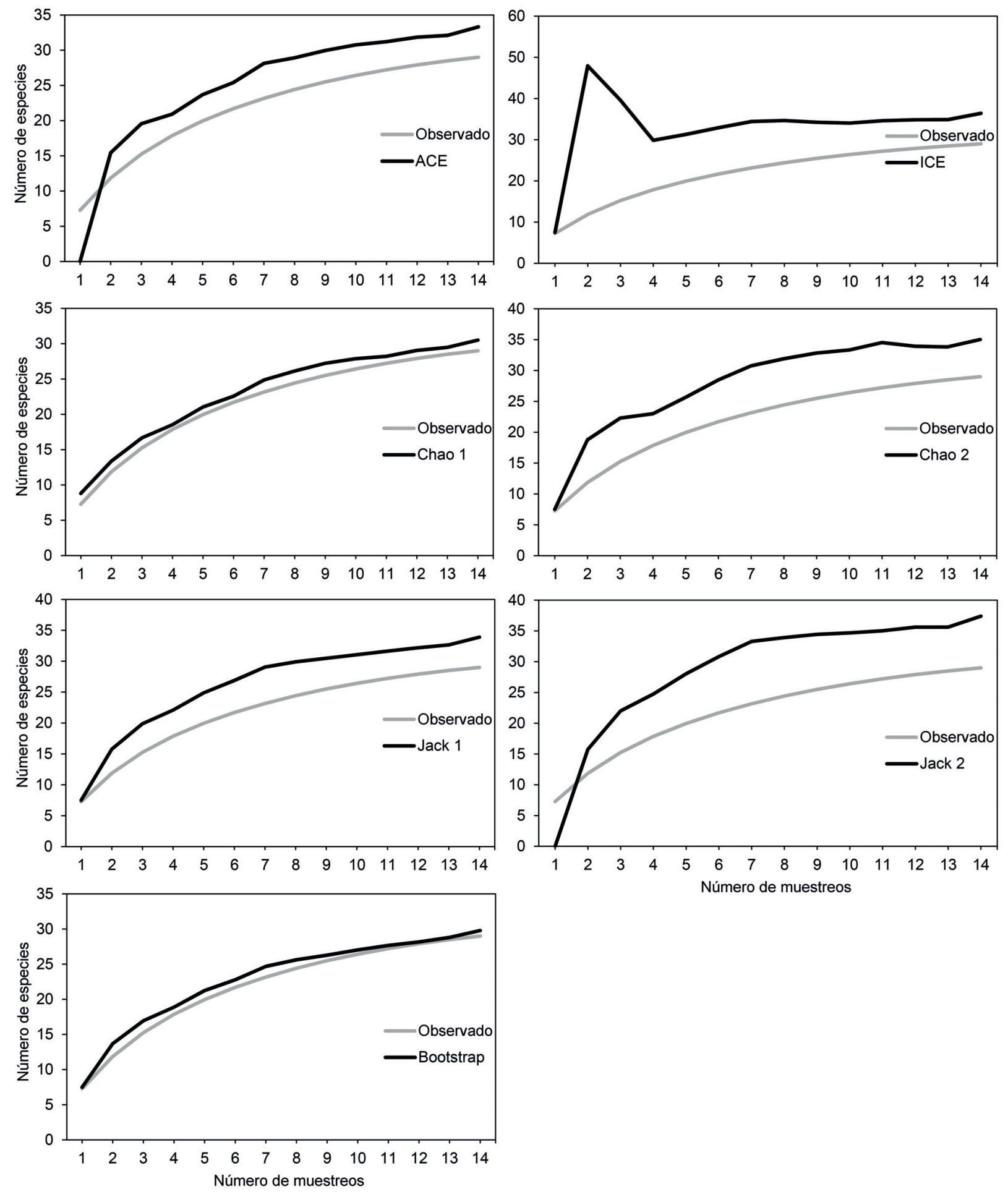

Figura 5. Curvas suavizadas de acumulación de especies de acuerdo a los estimadores no paramétricos ACE, ICE, Chao 1, Chao 2, Jack 1, Jack 2 y Bootstrap, para coleópteros epigeos de Isla Chañaral. / Smooth richness accumulation curves for the non-parametric estimators ACE, ICE, Chao 1, Chao 2, Jack 1, Jack 2 and Bootstrap, for epigean coleopterans from Chañaral Island. 


\section{DISCUSIÓN}

Diversos autores han mencionado la complejidad en la interpretación de los estimadores no paramétricos (Fogo et al. 2003; Fattorini et al. 2013a) así como también las limitantes que pueden presentar los distintos modelos asintóticos en la predicción del número teórico de especies para un área particular (González-Oreja et al. 2010). Sin embargo, a la fecha siguen siendo métodos bastante utilizados y su uso se extiende en diversos grupos de artrópodos, tanto insectos como arácnidos (Fattorini et al. 2013b; Zmihorski et al. 2013; Jiménez-Valverde et al. 2015; Wei et al. 2016).

El modelo de dependencia lineal, aplicado a los datos, mostró valores de riqueza teórica de especies cercanos a los observados en las islas Choros y Damas. Si bien el porcentaje de eficiencia de muestreo de este modelo mostró valores sobre el $90 \%$ e incluso sobre el $100 \%$ (ver Tabla 1); esto no indicaría que el total de las especies ha sido registrado mediante la metodología de muestreo utilizada en este estudio, sino que se sugiere realizar muestreos a escalas más finas de análisis (Escalante 2005). De acuerdo a Moreno \& Halffter (2000) este modelo llegaría a una asíntota más rápido en hábitats pequeños en comparación a paisajes heterogéneos más grandes. A diferencia de los modelos paramétricos, los estimadores no paramétricos evaluados mostraron valores de riqueza sobre los valores observados para todas las islas estudiadas. Para Isla Choros, los estimadores mejor evaluados en términos de sesgo y exactitud serían ICE y Chao 2, de los cuales sólo la curva de acumulación de ICE alcanzó una asíntota estable, lo que sugiere un inventario completo utilizando la metodología de muestreo del presente estudio. Tanto ICE como Chao 2 estarían sobreestimando la riqueza, mientras que este último sería más exacto al presentar una riqueza estimada cercana a la riqueza verdadera en Choros. Por otro lado, ICE sería el estimador menos sesgado y de mayor exactitud para Isla Damas, y sobreestimaría la riqueza para esta isla. Sin embargo, la curva de acumulación de este estimador mostró un rápido crecimiento, seguido de una caída con una posterior estabilidad sólo al aumentar el número de muestreos, lo que indicaría que este estimador es dependiente del número de muestras utilizadas y no de confiabilidad ante un bajo número de muestras (Rico-G et al. 2005). Esta condición se observó en todas las islas estudiadas.

De acuerdo a Toti et al. (2000) un buen estimador debería cumplir las siguientes características: (1) alcanzar la estabilidad con un bajo número de muestras de las requeridas para que la curva de acumulación de especies alcance la estabilidad, (2) su estimación no debe diferir en gran medida de otros estimadores y (3) su estimación deber ser cercana a una extrapolación visual razonable de la asíntota de la curva de acumulación de especies. Sin embargo, a excepción del estimador ICE, ningún estimador no paramétrico evaluado en este estudio alcanzó una asíntota definida y estable para las islas estudiadas, por lo que en futuros estudios proponemos analizar el comportamiento de la curva como un criterio fiable en la evaluación de estos estimadores.

Por otra parte, para Isla Chañaral el mejor modelo paramétrico evaluado (modelo de Clench) no alcanzó una asíntota clara, observándose una eficiencia de muestreo menor al 90\% (ver tabla 1), lo que indicaría la necesidad de mayores unidades de muestreo, considerando la alta heterogeneidad y mayor superficie de esta isla. De acuerdo a los parámetros de este modelo y siguiendo la ecuación presentada en Jiménez-Valverde \& Hortal (2003), se necesitarían 86 unidades de muestreo para alcanzar una eficiencia de muestreo cercana al 95\%, valor desproporcionadamente alto considerando la logística necesaria para muestrear estos ecosistemas insulares. Al utilizar modelos asintóticos, se hace complejo registrar nuevas especies a medida que se aumentan las unidades de muestreo y el inventario en campo (Jiménez-Valverde \& Hortal 2003), lo que obliga a evaluar la relación entre el esfuerzo adicional necesario (costo económico) y el grado de mejora en el inventario.

Mayores muestreos en otros hábitats particulares, aunque de difícil acceso en estas islas (e.g., mesetas, quebradas interiores, paleodunas y cavernas costeras), podrían aumentar y mejorar el registro de especies tal como lo señalan Pedraza et al. (2010). Es importante destacar en posteriores monitoreos la inclusión de otras estaciones del año y diferentes años ya que la composición de estos inventarios de especies puede variar de manera importante a lo largo del tiempo (Adler \& Lauenroth 2003). Esta evaluación en una escala temporal más amplia podría permitir registrar especies raras con ciclos biológicos particulares y/o distribuciones restringidas que no han sido registradas a la fecha por nuestros muestreos. La necesidad de un mayor número de muestreos dirigidos en esta área protegida tiene una real importancia desde el punto de vista del conocimiento taxonómico, el cual es un aspecto fundamental para ayudar a priorizar esfuerzos de conservación de la biota tanto local como global (Braby \& Williams 2016). En los últimos años diversos autores han documentado nuevas entidades taxonómicas de artrópodos para esta reserva (Pizarro-Araya \& Flores 2004; OjangurenAffilastro et al. 2007; Grismado \& Pizarro-Araya 2016), lo que refleja la importancia de estas islas como áreas que han promovido eventuales procesos de especiación locales. Esperamos que este trabajo por una parte represente la primera evaluación de los muestreos de coleópteros epigeos realizados en ecosistemas insulares del desierto costero de Chile, y por otra parte sirva como una herramienta para priorizar y dirigir futuros esfuerzos de muestreos que busquen aumentar los niveles de riqueza conocidos para esta área natural protegida. 


\section{AGRADECIMIENTOS}

A Mario Meléndez, Carla Louit, Paula Martínez, Pablo Arróspide y Cristian Rivera (CONAF) por la ayuda en los permisos y facilidades para trabajar en la Reserva Nacional Pingüino de Humboldt (Proyectos CONAF N. 006/2014 y 028/2015). Agradecemos a Luis Letelier (Centro de Investigación en Recursos Naturales y Sustentabilidad, Universidad Bernardo O'Higgins, Chile) por proporcionar asistencia en SIG y a los revisores anónimos cuyas sugerencias ayudaron a mejorar nuestro artículo. El presente estudio fue financiado por los proyectos FPA 04-015-2006 de CONAMA (Región de Coquimbo) y DIULS (PR15121 y PR17121/VACDDI001) de la Universidad de La Serena, La Serena, Chile (JPA).

\section{REFERENCIAS}

Adler, P.B., Lauenroth, W.K. 2003. The power of time: spatiotemporal scaling of species diversity. Ecology Letters 6(8):749-756.

Aguirre, L. 1967. Geología de las islas Choros, Damas y de Punta Choros, Provincia de Coquimbo. Revista Minerales (Chile) 22:73-83.

Alfaro, F.M., Pizarro-Araya, J., Flores, G.E. 2009. Epigean tenebrionids (Coleoptera: Tenebrionidae) from the Choros Archipelago (Coquimbo Region, Chile). Entomological News 120(2):125-130.

Alfaro, F.M., Pizarro-Araya, J., Mondaca, J. 2014. New insular records of Germarostes (Germarostes) posticus (Germar) (Coleoptera, Hybosoridae, Ceratocanthinae) for the Chilean transitional coastal desert. The Coleopterists Bulletin 68(3):387-390.

Alfaro, F.M., Pizarro-Araya, J., Flores, G.E. 2016. Composición y estructura del ensamble de tenebriónidos epigeos (Coleoptera: Tenebrionidae) de ecosistemas continentales e insulares del desierto costero transicional de Chile. Revista Mexicana de Biodiversidad 87:1283-1291.

Arancio, G., JARA, P. 2007. Flora de la Reserva Nacional Pingüino de Humbolt. Ediciones Universidad de La Serena, La Serena, Chile. 71 pp.

Armesto, J.J., Vidiella, P.E., Gutiérrez, J.R. 1993. Plant communities of the fog-free coastal desert of Chile: plant strategies in a fluctuating environment. Revista Chilena de Historia Natural 66:271-282.

Benítez, H.A., Pizarro-Araya, J., Bravi, R., Sanzana, M.J., Alfaro, F.M. 2014. Morphological variation on isolated populations of Praocis (Praocis) spinolai. Journal of Insect Science 14:11. doi:10.1673/031.014.11.

Braby, M.F., Williams, M.R. 2016. Biosystematics and conservation biology: critical scientific disciplines for the management of insect biological diversity. Austral Entomology 55(1):1-7.

Brose, U. 2002. Estimating species richness of pitfall catches by non-parametric estimators. Pedobiologia 46:101-107.

Brose, U., Martínez, N.D., Williams, R.V. 2003. Estimating species richness: sensitivity to sample coverage and insensitivity to spatial patterns. Ecology 84(9):2364-2377.

Castro, C., Brignardello, L. 2005. Geomorfología aplicada a la ordenación territorial de litorales arenosos. Orientaciones para la protección, usos y aprovechamientos sustentables del sector de Los Choros, Comuna de La Higuera, IV Región. Revista de Geografía Norte Grande 33:33-58.

Cepeda-Pizarro, J., Pizarro-Araya, J., Vásquez, H. 2005a. Composición y abundancia de artrópodos epigeos del Parque Nacional Llanos de Challe: impactos del ENOS de 1997 y efectos del hábitat pedológico. Revista Chilena de Historia Natural 78(4):635-650.

Cepeda-Pizarro, J., Pizarro-Araya, J., Vásquez, H. 2005 b. Variación en la abundancia de Arthropoda en un transecto latitudinal del desierto costero transicional de Chile, con énfasis en los tenebriónidos epigeos. Revista Chilena de Historia Natural 78(4):651-663.

Chao, A., Chiu, C.H. 2016. Nonparametric Estimation and Comparison of Species Richness. eLS 1-11.

Chazdon, R.L., Colwell, R.K., Denslow, J.S., Guariguata, M.R. 1998. Statistical methods for estimating species richness of woody regeneration in primary and secondary rain forests of Northeastern Costa Rica. En: Forest Biodiversity Research, Monitoring and Modeling: Conceptual Background and Old World Case Studies (Eds. Dallmeier, F. \& Comiskey, J.A.), pp. 285-309. The Parthenon Publishing Group, Paris.

Chiarucci, A., Enright, N.J., Perry, G.L., Miller, B.P., Lamont, B.B. 2003. Performance of nonparametric species richness estimators in a high diversity plant community. Diversity and Distributions 9(4):283-295.

Clarke, K.R., Warwick, R.M. 1998. A taxonomic distinctness index and its statistical properties. Journal of Applied Ecology 35:523-531.

Clench, H. 1979. How to make a regional list of butterflies: some throughts. Journal of the Lepidopteran Society 33:216-231.

Colwell, R.K. 2013. EstimateS: Statistical estimation of species richness and shared species from samples. Version 9. URL: purl.oclc.org/estimates.

Colwell, R.K., Coddington, J.A. 1994. Estimating terrestrial biodiversity through extrapolation. Philosophical Transactions of the Royal Society B: Biological Sciences 345(1311):101-118.

CONAF. 1997. Plan de manejo Reserva Nacional Pingüino de Humboldt. CONAF Regiones de Atacama y Coquimbo, Chile.

Cutko, A. 2009. Biodiversity Inventory of Natural Lands: A How-To Manual for Foresters and Biologists. Arlington, Virginia: NatureServe. $31 \mathrm{pp}$.

di Castri, F., HajeK, E.R. 1976. Bioclimatología de Chile. Imprenta-Editorial de la Universidad Católica de Chile. Santiago, Chile. 163 pp.

Elgueta, M., Marvaldi, A.E. 2006. Lista sistemática de las especies de Curculionoidea (Insecta: Coleoptera) presentes en Chile, con su sinonimia. Boletin del Museo Nacional de Historia Natural 55:113-153.

Escalante, T. 2003. ¿Cuántas especies hay?: Los estimadores no paramétricos de Chao. Elementos 52:53-56.

Escalante, T. 2005. Las bases de datos curatoriales y el estudio espacial de la biodiversidad: Un ejemplo con los mamíferos terrestres de México. En: Regionalización biogeográfica 
en Iberoamérica y tópicos afines: primeras jornadas biogeográficas de la Red Iberoamericana de Biogeografía y Entomología Sistemática. (Eds. Llórente, J. \& Morrone, J.J.), pp. 339-350. CYTED-UNAM-Conabio, México.

FATTORINI, S. 2013a. Faunistic knowledge and insect species loss in an urban area: the tenebrionid beetles of Rome. Journal of Insect Conservation 17(3):637-643.

FAtTORINI, S. 2013b. Regional Insect Inventories Require Long Time, Extensive Spatial Sampling and Good Will. PLoS ONE 8(4):e62118.

Flores, G.E., Pizarro-Araya, J. 2012. Systematic revision of the South American genus Praocis Eschscholtz, 1829 (Coleoptera: Tenebrionidae). Part 1: Introduction and subgenus Praocis s. str. Zootaxa 3336:1-35.

Flores, G.E., Pizarro-Araya, J. 2014. Towards a revision of the South American genus Praocis Eschscholtz (Coleoptera: Tenebrionidae), with estimation of the diversity of each subgenus. ZooKeys 415:53-80.

Foggo, A., Rundle, S.D., Bilton, D.T. 2003. The net result: evaluating species richness extrapolation techniques for littoral pond invertebrates. Freshwater Biology 48(10):1756-1764.

González-Oreja, J.A., de la Fuente-Díaz-Ordaz, A.A., Hernández-Santín, L., Buzo-Franco, D., BonacheRegidor, C. 2010. Evaluación de estimadores no paramétricos de la riqueza de especies. Un ejemplo con aves en áreas verdes de la ciudad de Puebla, México. Animal Biodiversity and Conservation 33(1):31-45

Gómez-Anaya, J.A., Novelo-Gutiérrez, R., Ramírez, A., ArcePérez, R. 2014. Using empirical field data of aquatic insects to infer a cut-off slope value in asymptotic models to assess inventories completeness. Revista Mexicana de Biodiversidad 85(1):218-227.

Gotelli, N.J., Colwell, R.K. 2001. Quantifying biodiversity: procedures and pitfalls in the measurement and comparison of species richness. Ecology Letters 4(4):379-391.

Gotelli, N.J., Colwell, R.K. 2011. Estimating species richness. En: Biological Diversity: Frontiers in Measurement and Assessment. (Eds. Magurran, A.E. \& McGill, B.J.), pp. 39-54. Oxford University Press: EUA.

Grismado, C.J., Pizarro-Araya, J. 2016. The spider genus Cyrioctea Simon on Isla Chañaral (Pingüino de Humboldt National Reserve, Atacama, Chile): Description of a new species, and description of the male of Cyrioctea cruz Platnick (Araneae, Zodariidae). Zootaxa 107:267-276.

JimÉneZ-VAlverde, A., Hortal, J. 2003. Las curvas de acumulación de especies y la necesidad de evaluar la calidad de los inventarios biológicos. Revista Ibérica de Aracnología 8:151-161.

Jiménez-Valverde, A., Gilgado, J.D., Sendra, A., Pérez-Suárez, G., Herrero-Borgoñón, J.J., Ortuño, V.M. 2015. Exceptional invertebrate diversity in a scree slope in Eastern Spain. Journal of Insect Conservation 19(4):713728.

Kulzer, H. 1959. Neue Tenebrioniden aus Südamerika (Col.) 18 Beitrag zur Kenntnis der Tenebrioniden. I Die Gattung Gyriosomus Guérin (Nycteliini). Entomologische Arbeiten Aus Dem Museum Georg Frey 10:523-547.

MagurRAn, A.E. 1988. Ecological diversity and its measurement. Princeton University Press, New Jersey. 179 pp.
Magurran, A.E. 2004. Measuring Biological Diversity. Blackwell, Malden. 264 pp.

Moreno, C.E., Halffter, G. 2000. Assessing the completeness of bat biodiversity inventories using species accumulation curves. Journal of Applied Ecology 37:149-158.

Moreno, C.E. 2001. Métodos para Medir la Biodiversidad. CYTED, RCYT/UNESCO y SEA, Zaragoza. 83 pp.

NovoA, R., VillasecA, S. 1989. Mapa agroclimático de Chile. Instituto de Investigaciones Agropecuarias, Santiago, Chile. 221 pp.

Ojanguren-Affilastro, A.A., Agusto, P., Pizarro-Araya, J., Mattoni, C.I. 2007. Two new scorpion species of genus Brachistosternus (Scorpiones: Bothriuridae) from northern Chile. Zootaxa 1623:55-68.

Palmer, M.W. 1990. The Estimation of Species Richness by Extrapolation. Ecology 71(3):1195-1198.

Pedraza, Ma. del Carmen, Márquez, J., Gómez-Anaya, J.A. 2010. Estructura y composición de los ensamblajes estacionales de coleópteros (Insecta: Coleoptera) del bosque mesófilo de montaña en Tlanchinol, Hidalgo, México, recolectados con trampas de intercepción de vuelo. Revista Mexicana de Biodiversidad 81(2):437-456.

PeÑA, L.E. 1971. Revisión del género Nycterinus Eschscholtz 1829 (Coleoptera: Tenebrionidae). Boletín del Museo Nacional de Historia Natural 32:129-158.

PeñA, L.E. 1974. Los tenebriónidos del género Thinobatis Esch. (Coleoptera: Tenebrionidae). Boletín del Museo Nacional de Historia Natural XLVIII: 243-252.

PeÑa, L.E. 1980. Aporte al conocimiento de los tenebriónidos de América del Sur (Coleoptera: Tenebrionidae). Revista Chilena de Entomología 10:37-59.

Pizarro-Araya, J., Flores, G.E. 2004. Two new species of Gyriosomus Guérin-Méneville from Chilean coastal desert (Coleoptera: Tenebrionidae: Nycteliini). Journal of The New York Entomological Society 112(2/3):121-126.

Pizarro-Araya, J., Vergara, O.E., Flores, G.E. 2012. Gyriosomus granulipennis (Coleoptera: Tenebrionidae) un caso extremo a conservar. Revista Chilena de Historia Natural 85(3):345-349.

Pizarro-Araya, J., Alfaro, F.M., Cortés-Contreras, M., Rivera, C., Vargas-Talciani, P., Ojanguren-Affilastro, A.A. 2014a. Epigean Insects of Chañaral Island (Pingüino de Humboldt National Reserve, Atacama, Chile). Journal of the Entomological Research Society 16(2):39-50.

Pizarro-Araya, J., Agusto, P., López-Cortés, F., OjangurenAffilastro, A.A., Briones, R., Cepeda-Pizarro, J. 2014 b. Diversidad y composición estacional de la escorpiofauna (Arachnida: Scorpiones) del archipiélago Los Choros (Región de Coquimbo, Chile). Gayana 78(1):46-56.

Pizarro-Araya, J., Alfaro, F.M., Flores, G.E., Letelier, L. 2017. Distribution and conservation status of Gyriosomus granulipennis Pizarro-Araya \& Flores 2004 (Coleoptera: Tenebrionidae). The Coleopterists Bulletin 71(4):1-6.

Rico-G, A., Beltrán, J.P., Alvarez, A., Florez, E. 2005. Diversidad de arañas (Arachnida: Araneae) en el Parque Nacional Natural Isla Gorgona, pacífico colombiano. Biota Neotropica 5:99-110.

SÁnchez-Fernández, D., Lobo, J.M., Abellán, P., Millán, A. 2011. How to identify future sampling areas when information is biased and scarce: An example using predictive models 
for species richness of Iberian water beetles. Journal for Nature Conservation 19(1):54-59.

Soberón, J., Llorente, J. 1993. The use of species accumulation functions for the prediction of species richness. Conservation Biology 7(3):480-488.

Solervicens, J. 2001. Clave para los géneros de Cleridae de Chile (Coleoptera). Acta Entomológica Chilena 25:41-46.

SoKal, R.R., RohlF, F.J. 1995. Biometry. Third edition. W. H. Freeman, New York, New York, USA. 884 pp.

StatSoft. 2011. STATISTICA (data analysis software system), version 10. URL: www.statsoft.com.

Toti, D.S., Coyle, F.A., Miller, J.A. 2000. A structured inventory of appalachian grass bald and heath bald spider assemblages and a test of species richness estimator performance. Journal of Arachnology 28:329-345.

Valdivia, D.E., Pizarro-Araya, J., Cepeda-Pizarro, J., OJangurenAffilastro, A.A. 2008. Diversidad taxonómica y densoactividad de solífugos (Arachnida: Solifugae) asociados a un ecosistema desértico costero del centro norte de Chile.
Revista de la Sociedad Entomológica Argentina 67(1-2):110.

Valdivia, D.E., Pizarro-Araya, J., Briones, R., OjangurenAffilastro, A.A., Cepeda-Pizarro, J. 2011. Taxonomical diversity and abundance of solpugids (Arachnida: Solifugae) in coastal ecotopes of north-central Chile. Revista Mexicana de Biodiversidad 82(4):1234-1242.

Walther, B.A., Moore, J.L. 2005. The concepts of bias, precision, and accuracy, and their use in testing the performance of species richness estimators, with a literature review of estimator performance. Ecography 28(6):1-15.

Wei, J., Niu, M., Feng, J. 2016. Diversity and Distribution Patterns of Scale Insects in China. Annals of the Entomological Society of America 109(3):405-414.

Zminorski, M., Sienkiewicz, P., Tryjanowski, P. 2013. Never ending story: a lesson in using sampling efficiency methods with ground beetles. Journal of Insect Conservation 17(2):333337.

Recibido: 03.08.2016

Aceptado: 10.07.2017 